\title{
Discrete Boltzmann modeling of unsteady reactive flows with nonequilibrium effects
}

\author{
Chuandong $\operatorname{Lin}^{1, *}$ and Kai H. Luo ${ }^{1,2, \dagger}$ \\ ${ }^{1}$ Center for Combustion Energy; Key Laboratory for Thermal Science and Power Engineering of Ministry of Education, \\ Department of Energy and Power Engineering, Tsinghua University, Beijing 100084, China \\ ${ }^{2}$ Department of Mechanical Engineering, University College London, Torrington Place, London WC1E 7JE, United Kingdom
}

(Received 12 June 2018; published 29 January 2019)

\begin{abstract}
A multiple-relaxation-time discrete Boltzmann model (DBM) is developed for compressible thermal reactive flows. A unified Boltzmann equation set is solved for hydrodynamic and thermodynamic quantities as well as higher order kinetic moments. The collision, reaction, and force terms are uniformly calculated with a matrix inversion method, which is physically accurate, numerically efficient, and convenient for coding. Via the Chapman-Enskog analysis, the DBM is demonstrated to recover reactive Navier-Stokes (NS) equations in the hydrodynamic limit. Both specific heat ratio and Prandtl number are adjustable. Moreover, it provides quantification of hydrodynamic and thermodynamic nonequilibrium effects beyond the NS equations. The capability of the DBM is demonstrated through simulations of chemical reactions in the free falling process, sound wave, thermal Couette flow, and steady and unsteady detonation cases. Moreover, nonequilibrium effects on the predicted physical quantities in unsteady combustion are quantified via the DBM. It is demonstrated that nonequilibrium effects suppress detonation instability and dissipate small oscillations of fluid flows.
\end{abstract}

DOI: 10.1103/PhysRevE.99.012142

\section{INTRODUCTION}

Reactive flows are ubiquitous in energy and environment systems and have received considerable attention [1]. They generally encompass a wide variety of nonlinear, unsteady, and nonequilibrium processes and are deemed complex due to the facts that the interplay between the chemical reaction and fluid flow is often significant, the chemical, hydrodynamic, and thermodynamic nonequilibrium influences are usually prominent, and the range of timescales involves several orders of magnitude [2]. To probe the dynamic process, the conventional simulation methods are based upon the continuum assumption, such as the Euler simulation [3], direct numerical simulation [4], large eddy simulation [5], Reynolds-averaged Navier-Stokes (NS) [6], etc. Continuum models could capture hydrodynamic nonequilibrium (such as the pressure, velocity, and temperature) as well as part of thermodynamic nonequilibrium (including the bulk viscosity, mass diffusion, and heat flux) coupled to the chemically reactive nonequilibrium [2]. Examples include the state-to-state vibrational model [7], multitemperature model [8,9], one-temperature model [10], etc. On the basis of NS models, the bulk viscosity coefficient and relaxation pressure have been studied [2,11], and it has been demonstrated that the bulk viscosity suppresses pressure oscillations [3].

Despite their success in reactive and nonreactive continuum flows, traditional hydrodynamic models may lack thermodynamic consistency where nonequilibrium and/or rarefaction effects play prominent roles [12,13], as in the porous medium [14], over the catalyst surface [15], across the

\footnotetext{
*chuandonglin@163.com

†K.Luo@ucl.ac.uk
}

multiphase interface [16], around the shock and detonation fronts $[17,18]$. To overcome this shortcoming, microscopic methods, such as nonequilibrium molecular dynamics [19] and direct simulation Monte Carlo [20], can ensure thermodynamic consistency, but their computational cost is usually prohibitive. As the central equation in kinetic theory, the Boltzmann equation offers the possibility to simulate complex nonequilibrium flows with a wide range of spatiotemporal scales in an effective and accurate way. In fact, macroscopic transport equations for nonequilibrium and/or rarefied flows could be derived from the Boltzmann equation at various levels of accuracy [21]. The Boltzmann equation is equivalent to an infinite list of coupled moment equations [21], but one model with more moment equations is closer to the Boltzmann equation [22]. A typical example is the R13 model of Struchtrup, which is beyond the Burnett and SuperBurnett equations and yields continuous shock structures at all Mach numbers [23]. As a promising kinetic method, the lattice Boltzmann model (LBM) has made remarkable progress during the past three decades [24-31]. Different from macroscopic transport equations with various nonlinear spatial derivatives, the lattice Boltzmann equation is in a uniformly linear form, and its algorithm is easy to code. However, most LBMs aim only to mimic traditional macroscopic governing equations and ignore a variety of thermodynamic nonequilibrium effects included in the Boltzmann equation.

Recently, as a variant of the standard LBM, the discrete Boltzmann model (DBM) addresses the above issues [32-36]. In addition to recovering the reactive macroscopic equations, the DBM contains essential hydrodynamic and thermodynamic nonequilibrium information beyond the former. Roughly speaking, DBMs can be classified into two categories. One is the single-relaxation-time (SRT) DBM [32-34], which is based on the original Bhatnagar-Gross-Krook model. 
The relaxation speeds of various thermodynamic processes are simply taken the same, which results in some defects, including the fixed Prandtl number $\operatorname{Pr}=1$. To remove the restriction, it needs to add an artificial term to modify the collision term in an SRT model [37]. The other is the multiplerelaxation-time (MRT) DBM [35,36], where the multiple relaxation rates can be independent or coupled together. This could optimize overall properties [38] and extend physical capabilities of the kinetic model, for example, an adjustable Prandtl number. The pioneering work on discrete Boltzmann modeling of combustion and detonation was an SRT scheme [32]. Later, MRT models were developed for reactive flows, and nonequilibrium characteristics were investigated in steady nonequilibrium detonation phenomena $[35,36]$. In this article, we propose an MRT DBM with the utilization of a matrix inversion method, which is more accurate, efficient, and robust than previous methods [35,36]. Morevoer, it is developed and adopted to investigate the various nonequilibrium effects on two-dimensional (2D) unsteady reactive flows.

\section{DISCRETE BOLTZMANN MODEL}

The DBM has a unified set of equations to describe the density, velocity, temperature, and higher order kinetic moments simultaneously, which take the form

$$
\frac{\partial \mathbf{f}}{\partial t}+\mathbf{v} \cdot \nabla \mathbf{f}=\mathbf{\Omega}+\mathbf{R}+\mathbf{F}+\mathbf{A}
$$

where $t$ is the time, $\mathbf{v}=\operatorname{diag}\left(\begin{array}{llll}\mathbf{v}_{1} & \mathbf{v}_{2} & \cdots & \mathbf{v}_{N}\end{array}\right)$ denotes the discrete velocity with $N=16, \mathbf{f}=\left(\begin{array}{llll}f_{1} & f_{2} & \cdots & f_{N}\end{array}\right)^{\mathrm{T}}$ represents the discrete distribution function, and $\boldsymbol{\Omega}, \mathbf{R}, \mathbf{F}$, and A stand for the collision, reaction, force, and additional terms in velocity space, respectively.

\section{A. Collision term}

The collision term accounts for the change rate of distribution function due to molecular collisions and takes the form

$$
\boldsymbol{\Omega}=-\mathbf{C}^{-1} \mathbf{S}\left(\mathbf{M}_{\mathbf{f}}-\mathbf{M}_{\mathbf{f}}^{\mathrm{eq}}\right),
$$

where $\quad \mathbf{M}_{\mathbf{f}}=\left(\begin{array}{llll}M_{f 1} & M_{f 2} & \cdots & M_{f 16}\end{array}\right)^{\mathrm{T}} \quad$ and $\quad \mathbf{M}_{\mathbf{f}}^{\mathrm{eq}}=$ $\left(\begin{array}{llll}M_{f 1}^{\mathrm{eq}} & M_{f 2}^{\mathrm{eq}} & \cdots & M_{f 16}^{\mathrm{eq}}\end{array}\right)^{\mathrm{T}}$ are the kinetic moment of the discrete distribution function and its equilibrium counterpart, respectively. $\quad \mathbf{S}=\operatorname{diag}\left(\begin{array}{llll}S_{1} & S_{2} & \cdots & S_{16}\end{array}\right)$ controls the relaxation speed of $\mathbf{M}_{\mathbf{f}}$ approaching $\mathbf{M}_{\mathbf{f}}^{\mathrm{eq}} \cdot \mathbf{C}$ is a $16 \times 16$ square matrix bridging the velocity and moment space, and $\mathbf{C}^{-1}$ is the inverse of $\mathbf{C}$, see Appendix A. For the purpose of recovering NS equations in the hydrodynamic limit, the discrete equilibrium distribution function $\mathbf{f}^{\mathrm{eq}}=\left(\begin{array}{llll}f_{1}^{\mathrm{eq}} & f_{2}^{\mathrm{eq}} & \cdots & f_{16}^{\mathrm{eq}}\end{array}\right)^{\mathrm{T}}$ has to satisfy the following relationship:

$$
\iint f^{\mathrm{eq}} \Psi d \mathbf{v} d \eta=\sum_{i} f_{i}^{\mathrm{eq}} \Psi_{i}
$$

with $\Psi=1, \mathbf{v},\left(\mathbf{v} \cdot \mathbf{v}+\eta^{2}\right), \mathbf{v v},\left(\mathbf{v} \cdot \mathbf{v}+\eta^{2}\right) \mathbf{v}, \mathbf{v v v},(\mathbf{v} \cdot \mathbf{v}$ $\left.+\eta^{2}\right) \mathbf{v v}, \quad$ correspondingly, $\quad \Psi_{i}=1, \mathbf{v}_{i},\left(\mathbf{v}_{i} \cdot \mathbf{v}_{i}+\eta_{i}^{2}\right)$, $\mathbf{v}_{i} \mathbf{v}_{i},\left(\mathbf{v}_{i} \cdot \mathbf{v}_{i}+\eta_{i}^{2}\right) \mathbf{v}_{i}, \mathbf{v}_{i} \mathbf{v}_{i} \mathbf{v}_{i},\left(\mathbf{v}_{i} \cdot \mathbf{v}_{i}+\eta_{i}^{2}\right) \mathbf{v}_{i} \mathbf{v}_{i}$, and the

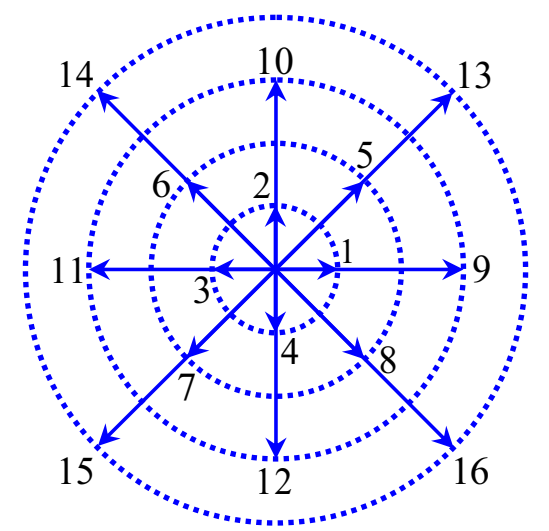

FIG. 1. Sketch for the discrete velocity model.

equilibrium distribution function [35]

$f^{\mathrm{eq}}=\frac{\rho}{2 \pi T}\left(\frac{1}{2 \pi I T}\right)^{1 / 2} \exp \left[-\frac{(\mathbf{v}-\mathbf{u}) \cdot(\mathbf{v}-\mathbf{u})}{2 T}-\frac{\eta^{2}}{2 I T}\right]$,

where $D=2$ denotes translational degrees of freedom, $I$ counts extra degrees of freedom due to vibration and/or rotation, and $\eta$ corresponds to vibrational and/or rotational energies.

In line with the conservation laws, $f_{i}^{\text {eq }}$ can be replaced by $f_{i}$ in Eq. (3) for $\Psi\left(\mathbf{v}_{i}\right)=1, \mathbf{v}_{i}$, and $\left(\mathbf{v}_{i} \cdot \mathbf{v}_{i}+\eta_{i}^{2}\right)$, from which the density $\rho$, velocity $\mathbf{u}$, and temperature $T$ are obtained. Substituting Eq. (4) into (3) leads to an explicit expression:

$$
\mathbf{M}_{\mathbf{f}}^{\mathrm{eq}}=\mathbf{C} \mathbf{f}^{\mathrm{eq}} \text {. }
$$

To ensure the matrix $\mathbf{C}$ invertible, a preferable discrete velocity form is the 2D 16-velocity (D2V16) model,

$$
\mathbf{v}_{i}=\left\{\begin{array}{lc}
\operatorname{cyc}: v_{a}( \pm 1,0), & 1 \leqslant i \leqslant 4 \\
\operatorname{cyc}: v_{b}( \pm 1, \pm 1), & 5 \leqslant i \leqslant 8 \\
\operatorname{cyc}: v_{c}( \pm 1,0), & 9 \leqslant i \leqslant 12 \\
\text { cyc }: v_{d}( \pm 1, \pm 1), & 13 \leqslant i \leqslant 16
\end{array}\right.
$$

with $\eta_{i}=\eta_{\alpha}, \eta_{b}, \eta_{c}$, and $\eta_{d}$ for $1 \leqslant i \leqslant 4,5 \leqslant i \leqslant 8,9 \leqslant$ $i \leqslant 12$, and $13 \leqslant i \leqslant 16$, respectively. Figure 1 delineates the sketch of D2V16.

It is noteworthy that the parameters $\left(v_{a}, v_{b}, v_{c}, v_{d}, \eta_{a}\right.$, $\left.\eta_{b}, \eta_{c}, \eta_{d}\right)$ are adjustable. To ensure numerical stability, their values can be adjusted to optimize the properties of the model: (1) The sizes of $v_{a}, v_{b}, v_{c}$, and $v_{d}$ should be around the values of flow velocity $\mathbf{u}$, sound speed $v_{s}=\sqrt{\gamma T}$, and shock speed, etc. (2) The sizes of $\eta_{a}, \eta_{b}, \eta_{c}$, and $\eta_{d}$ should be around the value of $\sqrt{I T}$. The reason for (1) is clear. The reason for (2) is as follows. In the thermodynamic equilibrium, $\frac{1}{2} m \bar{\eta}^{2}=$ $\frac{1}{2} I T$, where $m=1$ is the particle mass, and $\bar{\eta}^{2}$ represents the average value of $\eta^{2}$. Hence $\bar{\eta}^{2}=\sqrt{I T}$ is due to the equipartition of energy theorem, and the values of $\eta_{a}, \eta_{b}, \eta_{c}$, and $\eta_{d}$ should be around $\bar{\eta}$.

\section{B. Reaction term}

The reaction term is the variation rate of distribution function due to the chemical reaction. Its original expression reads 
[35]

$$
R=\frac{-(1+D) I T+I(\mathbf{v}-\mathbf{u})^{2}+\eta^{2}}{2 I T^{2}} f^{\mathrm{eq}} T^{\prime},
$$

with the varying rate of temperature $T^{\prime}=2 Q \lambda^{\prime} /(D+I)$, where the superscript' denotes the changing rate due to chemical reaction, $Q$ indicates the chemical heat release per unit mass of fuel, and $\lambda$ the mass fraction of chemical product. Its discrete form, $R_{i}$, satisfies the relation

$$
\iint R \Psi d \mathbf{v} d \eta=\sum_{i} R_{i} \Psi_{i}
$$

where the elements of $\Psi$ and $\Psi_{i}$ are the same as those in Eq. (3). Substituting Eq. (7) into Eq. (8) results in $\mathbf{M}_{\mathbf{R}}=\mathbf{C R}$ :

$$
\mathbf{R}=\mathbf{C}^{-1} \mathbf{M}_{\mathbf{R}},
$$

where $\mathbf{R}=\left(\begin{array}{llll}R_{1} & R_{2} & \cdots & R_{16}\end{array}\right)^{\mathrm{T}}$ and $\mathbf{M}_{\mathbf{R}}=\left(\begin{array}{ll}M_{R 1} & M_{R 2}\end{array}\right.$ $\left.\cdots M_{R 16}\right)^{\mathrm{T}}$ correspond to the reaction term in velocity and moment spaces, respectively, see Appendix A.

To mimic the essential dynamics of a chain-branching reaction [39], we employ a two-step reaction scheme,

$$
\begin{gathered}
\xi^{\prime}=H k_{I} \exp \left[E_{I}\left(T_{s}^{-1}-T^{-1}\right)\right], \\
\lambda^{\prime}=(1-H) k_{R}(1-\lambda) \exp \left(-E_{R} T^{-1}\right),
\end{gathered}
$$

where $H=1$ for $\xi<1$, otherwise, $H=0$. $\xi$ denotes the reaction progress variable in a thermally neutral induction period. $k_{I}$ and $k_{R}$ are the rate constants, and $E_{I}$ and $E_{R}$ are activation energies, for the ignition and reaction processes, respectively. $T_{s}$ is the temperature after the preshock wave. For the sake of simplicity, ionization, heat radiation, active radicals, and reversible reactions are ignored. Detailed or reduced chemical reaction mechanisms could also be studied and utilized with the kinetic DBM, but this is beyond the scope of this paper.

\section{Force term}

The force term is the variation rate of the distribution function due to the external force. Its original formula is specified as

$$
F=-\mathbf{a} \cdot \frac{\partial f^{\mathrm{eq}}}{\partial \mathbf{v}}=\frac{\mathbf{a} \cdot(\mathbf{v}-\mathbf{u})}{T} f^{\mathrm{eq}} .
$$

Its discrete form, $F_{i}$, satisfies

$$
\iint F \Psi d \mathbf{v} d \eta=\sum_{i} F_{i} \Psi_{i}
$$

where the elements of $\Psi$ and $\Psi_{i}$ are identical to those in Eqs. (3) and (8). Substituting Eq. (12) into Eq. (13) leads to $\mathbf{M}_{\mathbf{F}}=\mathbf{C F}$,

$$
\mathbf{F}=\mathbf{C}^{-1} \mathbf{M}_{\mathbf{F}},
$$

where $\quad \mathbf{F}=\left(\begin{array}{llll}F_{1} & F_{2} & \cdots & F_{16}\end{array}\right)^{\mathrm{T}} \quad$ and $\quad \mathbf{M}_{\mathbf{F}}=$ $\left(\begin{array}{llll}M_{F 1} & M_{F 2} & \cdots & M_{F 16}\end{array}\right)^{\mathrm{T}}$ are expressions of the force term in velocity and moment spaces, respectively, see Appendix A.

\section{Additional term}

Via the Chapman-Enskog expansion, it is found that one needs to modify the collision term with an additional term,

$$
\mathbf{A}=\mathbf{C}^{-1} \mathbf{M}_{\mathbf{A}}
$$

to recover the traditional NS equations. Here $\mathbf{M}_{\mathbf{A}}=$ $\left(\begin{array}{llllllll}0 & \cdots & 0 & M_{A 8} & M_{A 9} & 0 & \cdots & 0\end{array}\right)^{\mathrm{T}}$ is the kinetic moment of the additional term, with

$$
\begin{aligned}
& M_{A 8}=2\left(S_{8}-S_{5}\right)\left(u_{x} M_{f 5}^{\mathrm{neq}}+S_{5}^{-1} S_{6} u_{y} M_{f 6}^{\mathrm{neq}}\right), \\
& M_{A 9}=2\left(S_{9}-S_{7}\right)\left(u_{y} M_{f 7}^{\mathrm{neq}}+S_{6} S_{7}^{-1} u_{x} M_{f 6}^{\mathrm{neq}}\right),
\end{aligned}
$$

in terms of

$$
\begin{gathered}
M_{f 5}^{\mathrm{neq}}=\frac{2 \rho T}{S_{5}}\left(\frac{1-D-I}{D+I} \frac{\partial u_{x}}{\partial x}+\frac{1}{D+I} \frac{\partial u_{y}}{\partial y}\right), \\
M_{f 6}^{\mathrm{neq}}=-\frac{\rho T}{S_{6}}\left(\frac{\partial u_{x}}{\partial y}+\frac{\partial u_{y}}{\partial x}\right), \\
M_{f 7}^{\mathrm{neq}}=\frac{2 \rho T}{S_{7}}\left(\frac{1}{D+I} \frac{\partial u_{x}}{\partial x}+\frac{1-D-I}{D+I} \frac{\partial u_{y}}{\partial y}\right) .
\end{gathered}
$$

Let us explain the physical reason for the above modification. In Eq. (2) the relaxation coefficient $S_{i}$ appears independently related to each kinetic model $M_{f i}^{\mathrm{neq}}=M_{f i}-M_{f i}^{\mathrm{eq}}$, where $M_{f i}$ and $M_{f i}^{\mathrm{eq}}$ are elements of $\mathbf{M}_{\mathbf{f}}$ and $\mathbf{M}_{\mathbf{f}}^{\mathrm{eq}}$, respectively. However, in reality, there may be some physical associations between various relaxation processes. To ensure that our DBM could correctly capture the hydrodynamic behavior, we carry out the Chapman-Enskog analysis and compare the recovered macroscopic equations with traditional governing equations. Then the additional term is found as a solution to the difference between them. Hence, this modification aims to recover the consistent NS equations in the hydrodynamic limit, see Appendix B.

In addition, the DBM contains more detailed nonequilibrium effects than a traditional NS model. To be specific, $M_{f i}^{\text {neq }}$ is zero for $1 \leqslant i \leqslant 4$ due to the conservation laws, otherwise it may be nonzero in nonequilibrium systems. It can be found in Eq. (2) that the influence of $M_{f i}^{\text {neq }}$ is amplified by the factor $S_{i}$. Consequently $S_{i}$ has no influence for $1 \leqslant i \leqslant 4$, and it enhances (reduces) the nonequilibrium effects for $i \geqslant 5$ when $S_{i}$ is large (small). Note that the parameters $S_{5}=S_{6}=$ $S_{7}=S_{\mu}$ are related to the viscosity, and $S_{8}=S_{9}=S_{\kappa}$ are relevant to the thermal conductivity. Moreover, compared with traditional NS models that cannot describe well the rarefied effects of microscopic gas flows, it is convenient to have a proper kinetic boundary condition for the DBM to capture the velocity slip and flow characteristics in the Knudsen layer [40].

It is worth mentioning that the above matrix inversion method is a well-established approach in the scientific community dealing with the MRT LBM $[38,41]$. The mapping between moment space and discrete velocity space is linked by the transformation $\mathbf{C}$. From their expressions in moment space, not only the collision term but also extra source terms (including the force and heat source terms) can be obtained [41]. However, only the collision term is transformed from moment space into velocity space in previous DBMs 
[35,36,42]. In the current DBM, the collision, reaction, force, and additional terms are all calculated with the matrix inversion method. The processes of particle collision, chemical reaction, and acceleration are executed in moment space. This methodology has the following threefold advantages.

(1) High computational efficiency. The number of discrete distribution functions equals the number of discrete velocities, which is no less than the number of moment relations of the equilibrium discrete distribution function [43]. Additionally, a required number of moment relations of the force or reaction term should be satisfied in order to recover hydrodynamic equations and capture nonequilibrium effects. To this end, 24 discrete velocities are employed for the DBM with D2V24 in Ref. [35]. Obviously our DBM using D2V16 is more efficient.

(2) High physical and numerical accuracy. In the present model, 16 sets of kinetic moments calculated by summations of the discrete equilibrium discrete distribution function, force or reaction term are exactly equal to those calculated by integrals of their original forms. In comparison, only the first nine sets of moment relations of the reaction term are satisfied in Ref. [35]. As for the model in Ref. [36], the summations approach the corresponding integrals with only first-order temporal accuracy. Consequently the current method is physically more accurate and numerically more precise, which is of particular importance when measuring nonequilibrium effects.

(3) Unified mathematical algorithm. The DBM has the merit of simplicity of the algorithm and coding due to its unified formulation. Different from previous models where the collision, force, and reaction terms are expressed in various forms $[17,18,32,33,35,36]$, our DBM is more convenient to code as all terms are specified with the matrix inversion method in a uniform way. Furthermore, it is straightforward to extend this methodology to other physical mechanisms, such as the surface tension, electromagnetic action, etc.

\section{SIMULATION AND INVESTIGATION}

In this section, the versatile DBM is validated from several aspects, using benchmarks of chemical reactions in the free falling process, sound wave, thermal Couette flow, and steady and unsteady detonation. We confirm the following: (1) The DBM is suitable for subsonic, sonic and supersonic flows. (2) The effects of chemical reaction and/or external force are naturally coupled to the flow field. (3) Both specific heat ratio and Prandtl number are adjustable. (4) The DBM has the capability of measuring the nonequilibrium manifestations accurately. Furthermore, this model is employed to investigate nonequilibrium effects on unsteady detonation. Here the second-order Runge-Kutta scheme is adopted for the time derivative in Eq. (1), and the second-order nonoscillatory and nonfree-parameter dissipation difference scheme is employed for the space derivatives [44].

\section{A. Chemical reactions in the free falling process}

First, let us verify the effects of the external force and chemical reaction incorporated by the force and reaction terms, respectively. To this aim, we simulate the exothermic chemical reaction in a free falling box. Initially the box is
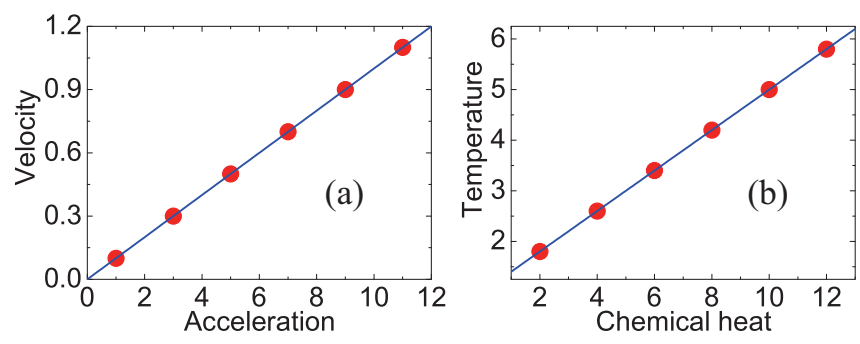

FIG. 2. Exothermic chemical reaction in the free falling process. (a) Velocity versus acceleration; (b) temperature versus chemical heat.

filled with a chemical reactant with density $\rho_{0}=1$, velocity $\mathbf{u}_{0}=0$, and temperature $T_{0}=1$. The field is uniform, and hence only one grid mesh, $N_{x} \times N_{y}=1 \times 1$, is adopted for the computational domain, and the periodic boundary condition is employed in $x$ and $y$ directions, respectively. The spatial and temporal steps are $\Delta x=\Delta y=10^{-3}$ and $\Delta t=$ $10^{-4}$, respectively. Relaxation parameters are $S_{i}=10^{3}$ and reaction parameters are $k_{I}=5 \times 10^{2}, E_{I}=8, k_{R}=10^{4}$, and $E_{R}=1$.

Figure 2(a) displays the vertical velocity $u_{y}$ versus acceleration $\mathbf{a}=a_{y} \mathbf{e}_{y}$, with fixed chemical heat $Q=12$, at time $t=0.1$ in the free falling process. Figure 2(b) illustrates the temperature $T$ versus chemical heat $Q$, with fixed acceleration $a_{y}=1$, after the chemical reaction. It is evident that our simulation results agree well with the exact solutions $\mathbf{u}=\mathbf{a} t$ in Fig. 2(a) and $T=T_{0}+(\gamma-1) Q$ in Fig. 2(b), respectively. Here $\gamma=(D+I+2) /(D+I)$ is the specific heat ratio. Consequently the matrix inversion method for the calculation of the force and reaction terms is accurate.

\section{B. Sound wave}

Now let us demonstrate that the DBM is capable of capturing the sound wave. The field is in a tube with density $\rho_{0}=1$ and velocity $\mathbf{u}_{0}=0$; see Fig. 3 . For the computational domain, the grid mesh is $N_{x} \times N_{y}=1000 \times 1$, and the spatial step is $\Delta x=\Delta y=10^{-3}$. The temporal step is $\Delta t=10^{-4}$. The outflow boundary condition is adopted in the $x$ direction, and the periodic boundary condition is employed in the $y$ direction. A small perturbation is initially imposed on the location $x_{0}=0.1$. Then the perturbation divides into two sound waves propagating forward and backward, respectively. Figure 4 exhibits the position of the sound wave versus time. Figure 4(a) is for two different specific heat ratios $\gamma=$ 1.2, 2.0 and fixed temperature $T=1.0$; Fig. 4(b) is for a different temperature $T=1.0,2.0$ and a fixed specific heat ratio $\gamma=1.2$. The sound speed is the function of the specific heat ratio and temperature. It is evident that the simulation results coincide well with the theoretical value $x=x_{0}+v_{s} t$.

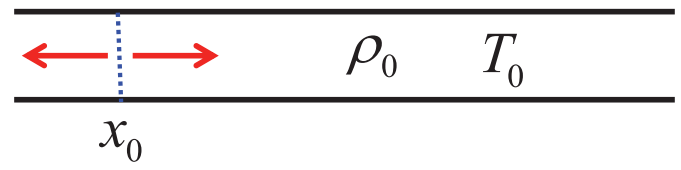

FIG. 3. Initial configuration for the sound wave simulation. 

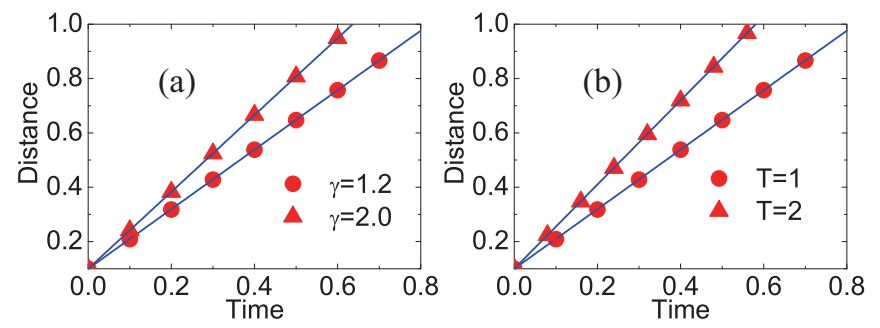

FIG. 4. Propagation of the sound wave: (a) with various specific heat ratios; (b) with various temperatures.

Consequently it is confirmed that our model has the capability of capturing the sound wave under various temperature and specific heat ratios.

\section{Thermal Couette flow}

In this subsection, the simulation of Couette flow is carried out for two purposes. One is to validate that the DBM is suitable for subsonic flow. The other is to demonstrate that the DBM is applicable to thermal flow with a flexible Prandtl number. As shown in Fig. 5, the initial state of the fluid is $\rho_{0}=1, T_{0}=1, \mathbf{u}_{0}=0$. Below the flow is a wall at rest with temperature $T_{1}=1$; above the flow is a wall moving leftwards with constant speed $u_{2}=0.1$ and temperature $T_{2}=1.001$. The height between the walls is $H=0.1$. The grid mesh is $N_{x} \times N_{y}=1 \times 100$, and the temporal step $\Delta t=10^{-4}$. Periodic boundary conditions are applied to the left and right boundaries, and the nonequilibrium extrapolation method is employed for the bottom and top boundaries.

Theoretically the distribution of temperature in the $y$ direction follows

$$
T=T_{1}+\left(T_{2}-T_{1}\right) \frac{y}{H}+\frac{\mu}{2 \kappa} u_{0}^{2} \frac{y}{H}\left(1-\frac{y}{H}\right)
$$

when the flow achieves a steady state. Here $\mu$ and $\kappa$ are the dynamic viscosity and thermal conductivity, respectively. The comparison between the simulation results and the analytical solutions is displayed in Fig. 6. The collision parameters are $S_{i}=10^{3}$, except $S_{5}=S_{6}=S_{7}=2 \times 10^{3}, 10^{3}$, and $5 \times 10^{2}$, for $\operatorname{Pr}=0.5,1.0$, and 2.0, respectively. It is clear that our simulation results agree well with the analytical solutions for various Prandtl numbers.

For the purpose of demonstrating that the DBM has the capability of capturing the nonequilibrium effects in the iterative process dynamically, we examine the nonequilibrium quantity $M_{f 6}^{\mathrm{neq}}=M_{f 6}-M_{f 6}^{\mathrm{eq}}$ in the case with $\operatorname{Pr}=0.5$. Figure 7 plots the DBM results (symbols) and the correspond-

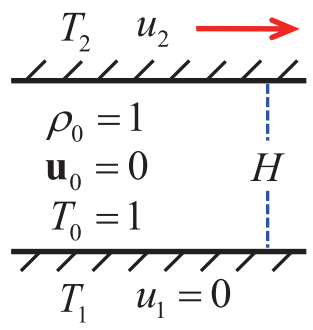

FIG. 5. Initial configuration for the Couette flow.

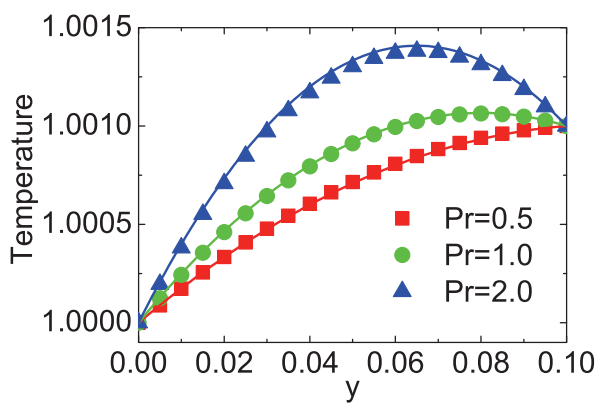

FIG. 6. Temperature profiles of Couette flow. The squares, circles, and triangles denote $\operatorname{Pr}=0.5,1.0$, and 2.0 , respectively.

ing analytic solutions (lines) at time instants $t=1.0,2.0$, and 4.0, respectively. Obviously they coincide well with each other.

\section{Steady detonation}

From numerical and physical points of view, denotations have different challenges from those of flames that are weak deflagrations. The reasons are as follows: (1) The compressibility and nonequilibrium effects exert a strong influence on a detonation system, where physical gradients are extremely sharp. (2) The values of relative velocities, ranging from subsonic, to sonic, and further to supersonic speed, have a wide span in a detonation, where the Mach number is greater than 1 . (3) The thermal properties have a great variation around a detonation wave, where the temperature and sound speed change considerably. Physically the particle velocity distribution and its local equilibrium vary significantly around the detonation front. Mathematically the discrete (equilibrium) distribution function changes greatly, and it is different to solve the stiff and oscillatory problems in the simulation of a detonation wave.

To demonstrate the robustness and accuracy of our model, we simulate a one-dimensional (1D) steady detonation here. Satisfying the Rankine-Hugoniot conditions, the initial configuration is

$$
\begin{aligned}
\left(\rho, u_{x}, u_{y}, T, \xi, \lambda\right)_{L} & =(1.6736,-3.8330,0,10.494,1,1), \\
\left(\rho, u_{x}, u_{y}, T, \xi, \lambda\right)_{R} & =(1,-6.4150,0,1,0,0),
\end{aligned}
$$

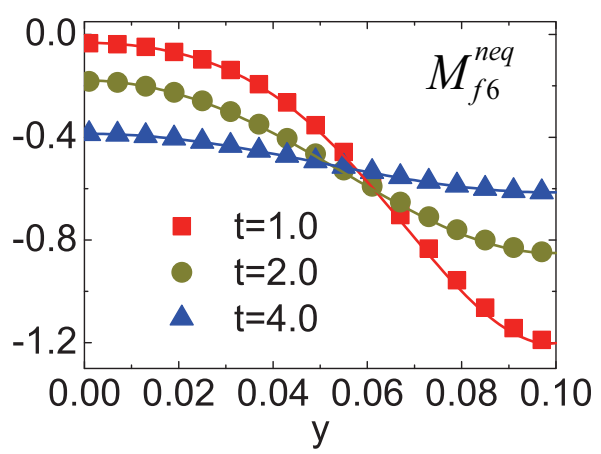

FIG. 7. Vertical distribution of $M_{f 6}^{\text {neq }}$ at time instants $t=$ 1.0, 2.0, and 4.0, respectively. Symbols denote the DBM results, lines denote the corresponding theoretical solutions. 

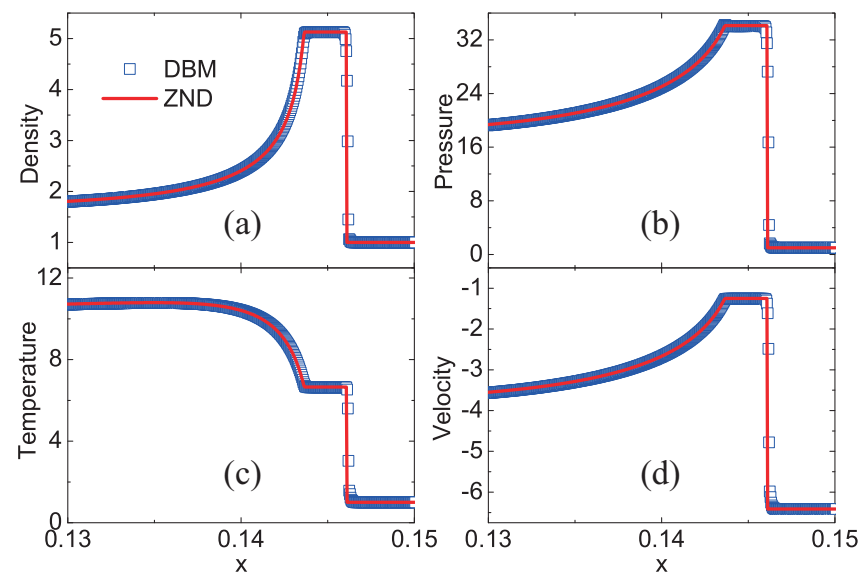

FIG. 8. Physical quantities around the detonation wave: (a) density, (b) pressure, (c) temperature, and (d) horizontal velocity. The squares represent the DBM results, the solid lines represent ZND solutions [1].

where the subscript $L$ indicates $0 \leqslant x \leqslant 0.18$, and $R$ indicates $0.18<x \leqslant 0.2$. The Mach number is 5.4217. To ensure the resolution is high enough, the grid is chosen as $N_{x} \times N_{y}=$ $5000 \times 1$, the space step $\Delta x=\Delta y=4 \times 10^{-5}$, and the time step $\Delta t=2 \times 10^{-6}$. The collision parameters are $S_{5}=S_{6}=$ $S_{7}=2 \times 10^{5}$, and $S_{i}=2.5 \times 10^{5}$ for the others. The specificheat ratio is $\gamma=1.5$. The reaction parameters are $Q=$ $20, k_{I}=5 \times 10^{2}, k_{R}=10^{3}, E_{I}=8, E_{R}=1$. In addition, inflow and outflow boundary conditions are employed in the $x$ direction, and the periodic boundary condition is adopted in the $y$ direction.

Figure 8 displays the detonation profiles: (a) density, (b) pressure, (c) temperature, and (d) horizontal velocity, at time $t=1$. The squares stand for the DBM results, and the solid lines stand for the solutions of the Zeldovich-Neumann-Doering (ZND) theory [1]. After the detonation wave, the quantities given by the current model are $\left(\rho, u_{x}, u_{y}, T\right)=(1.6738,-3.8340,0,10.489)$. Compared with the analytic solutions [1], the relative errors are $(0.01 \%, 0.03 \%, 0,0.05 \%)$, respectively. Obviously the DBM results agree well with the ZND solutions [1].

Figure 9 plots the comparison between the present DBM and two previous models [35,36]. The DBM in Ref. [35] produces nonphysical oscillations, which are soon amplified and result in numerical divergence. The present DBM and the DBM in Ref. [36] have obtained remarkably smooth profiles, which demonstrate their numerical robustness. Meanwhile, for the sake of comparison of their computational efficiency, we take a record of runtimes of the three models. Simulations are carried out on a personal computer with Intel Core i7-6700K CPU at 4.00 GHz, and RAM 32.0 GB. The installed 64-bit version system is capable of performing double-precision floating-point operations. The program is written in Fortran90, compiled using the Microsoft Visual Studio in version 2013, and run on a single processor. It takes $75 \mathrm{sec}$ to run the above program using the current model, while it requires $122 \mathrm{sec}$ for the DBM in Ref. [35] and 79 sec for the DBM in Ref. [36]. Obviously the current model has higher computational efficiency than previous models $[35,36]$.

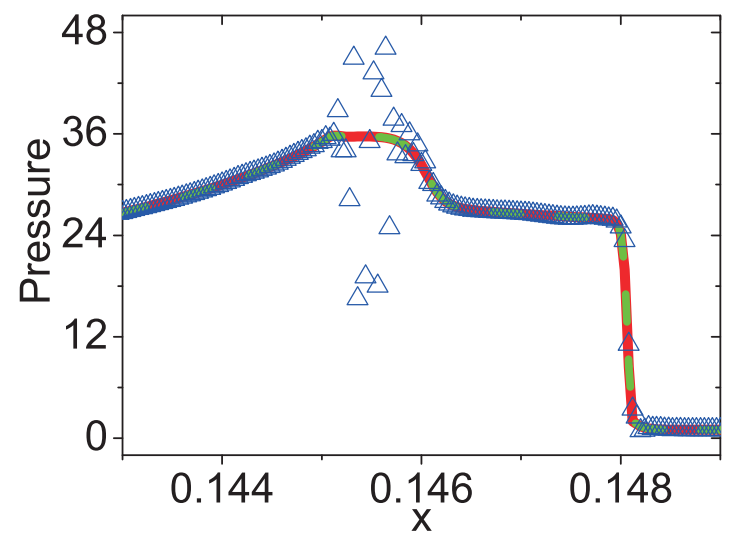

FIG. 9. Pressure around the detonation wave at time $t=1.7 \times$ $10^{-2}$. The solid line represents the current DBM, the triangles represent the DBM in Ref. [35], and the dashed line represents the DBM in Ref. [36].

Let us explain the reasons. Declare the discrete (equilibrium) distribution function as

$$
\begin{aligned}
& \text { REAL } * 8 \mathrm{f}(\mathrm{NI}, 1-\mathrm{NG}: \mathrm{NX}+\mathrm{NG}, 1 \\
& \quad-\mathrm{NG}: \mathrm{NY}+\mathrm{NG}), \\
& \mathrm{REAL} * 8 \text { feq(NI, } 1-\mathrm{NG}: \mathrm{NX}+\mathrm{NG}, 1 \\
& \quad-\mathrm{NG}: \mathrm{NY}+\mathrm{NG}),
\end{aligned}
$$

where the virtual grid is $\mathrm{NG}=2$, the grid mesh is $\mathrm{NX} \times$ $\mathrm{NY}=N_{x} \times N_{y}$, and the number of discrete velocities is $\mathrm{NI}=$ $N_{i}$. To be specific, $N_{i}=24$ for the DBM in Ref. [35], and $N_{i}=16$ for the current model and the one in Ref. [36]. The number of cycles within a blocked code snippet relevant to the discrete (equilibrium) distribution function is $N_{c}=N_{i} \times$ $N_{x} \times N_{y}$. Consequently the runtime of the 24-velocity model is approximately 1.5 times that of the 16-velocity model. Moreover, with the method used in Ref. [36], it needs to compute the equilibrium distribution functions before and after the chemical reaction. The difference between them is used to calculate the reaction term [36]. While the equilibrium distribution function is not required in the main loop for the current model, it is more efficient than Ref. [36].

\section{E. Unsteady detonation}

In addition to steady detonations, the DBM has the capability to describe unsteady detonations. The bifurcation boundary between steady and unsteady detonations depends on the ratio of the length of the heat release layer to that of the induction zone layer [39]. Let us consider unsteady detonations with the rate constant $k_{R}$ tunable and the other parameters fixed the same as the above steady detonation. Figure 10(a) exhibits the evolution of shock pressure in three cases with $k_{R}=$ 5000, 7500, and 10 000, respectively. In contrast, Fig. 10(c) plots the above three cases with the nonequilibrium terms switched off (see the Supplemental Material for more details about unsteady detonations [45]). To roughly estimate the deviation amplitude from equilibrium, we introduce the "nonequilibrium strength" $\Delta=\sqrt{\sum M_{f i}^{\text {neq2 }}}$. We further define the pressure amplitude as the distance between its crest and 


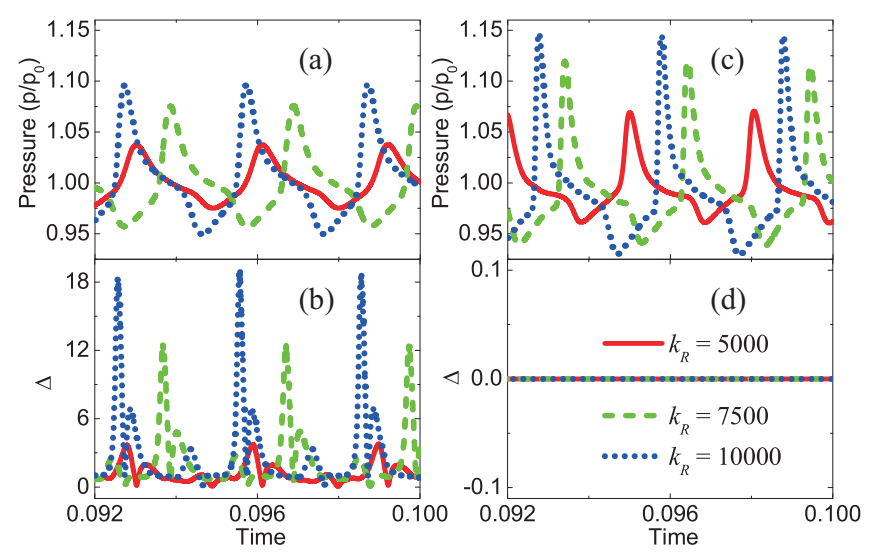

FIG. 10. (a) Shock pressure and (b) nonequilibrium strength, with nonequilibrium effects; (c) shock pressure and (d) nonequilibrium strength, without nonequilibrium effects. The solid, dashed, and dotted lines denote $k_{R}=5000,7500$, and 10000 , respectively.

trough. Figure 10(b) illustrates the nonequilibrium strength corresponding to Fig. 10(a), and Fig. 10(d) corresponds to Fig. 10(c).

It is evident in Fig. 10 that the unsteady detonations, with or without nonequilibrium effects, evolve periodically. To be specific, in Fig. 10(a), the amplitudes of $p / p_{0}$ are $0.06,0.12$, and 0.15 , for the cases with $k_{R}=5000,7500$, and 10000 , respectively. Here $p_{0}$ represents the shock pressure of the steady detonation. In Fig. 10(b), the amplitudes are $0.11,0.18$, and 0.22 , for the three cases, respectively.
Physically, with increasing $k_{R}$, the chemical reaction becomes more violent, and the physical oscillation is amplified. Meanwhile, the nonequilibrium effects are stronger for a higher reaction rate and sharper physical gradients [17]. Moreover, the amplitudes considering the nonequilibrium effects are smaller than those without nonequilibrium effects. Physically the increasing diffusion smooths the density gradient, and the increasing thermal conduction smooths the temperature gradient. Consequently the pressure amplitude is smaller when considering nonequilibrium effects, including the diffusion and thermal conduction [36].

Moreover, a 2D unsteady detonation is simulated, with the initial configuration,

$$
\begin{aligned}
& \left(\rho, u_{x}, u_{y}, T, \xi, \lambda\right)_{L}=(1.4804,-1.6995,0,2.0631,1,1), \\
& \left(\rho, u_{x}, u_{y}, T, \xi, \lambda\right)_{R}=(1,-2.5160,0,1,0,0),
\end{aligned}
$$

where $L$ indicates $0 \leqslant x \leqslant 0.18$, and $R$ indicates $0.18<x \leqslant$ 0.2 . Between the two parts is a sinusoidal perturbed interface with initial amplitude $A_{0}=2 \times 10^{-4}$. The parameters are $N_{x} \times N_{y}=1500 \times 500, \Delta x=\Delta y=2 \times 10^{-5}, \Delta t=1 \times 10^{-6}$, $Q=2, \quad\left(v_{a}, v_{b}, v_{c}, v_{d}, \eta_{a}, \eta_{b}, \eta_{c}, \eta_{d}\right)=(4,3.6,2.2$, $0.7,0,0,0,2.6)$, and the others are the same as those for the case $k_{R}=10000$ in Fig. 10. Figure 11 depicts the snapshots of the reaction process $\lambda$, the pressure $p$, and the nonequilibrium effect $\Delta$ at various times in the evolution of the 2D unsteady detonation (see the Supplemental Material for more details of unsteady detonations [45]). It can be found that the fields at the time $t=8.92 \times 10^{-2}$ are almost the same as those at the time $t=9.78 \times 10^{-2}$, which indicates

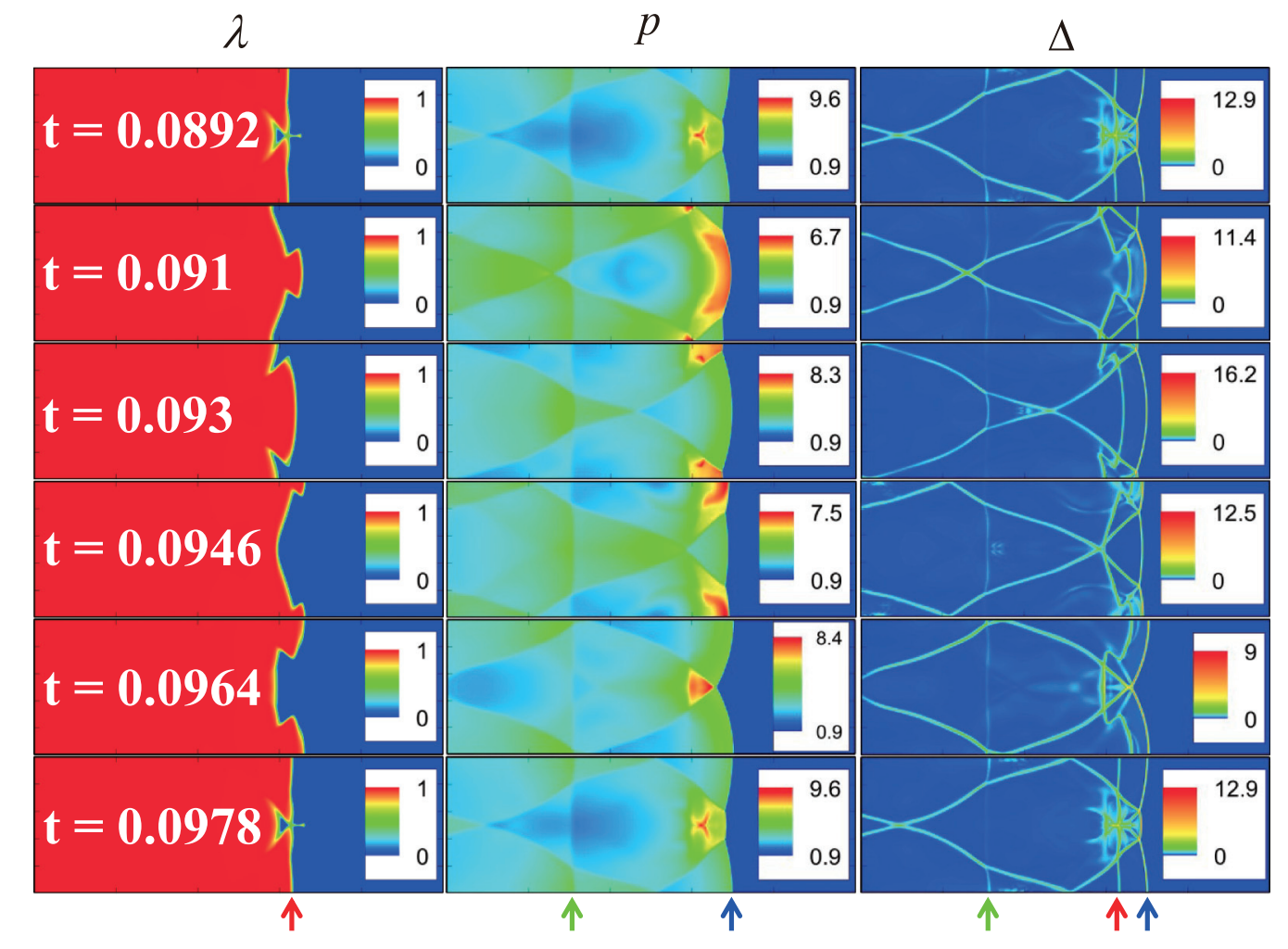

FIG. 11. Snapshots of the reaction process (left), pressure (middle), and nonequilibrium effect (right), during a period of the unsteady detonation from top to bottom, respectively. 

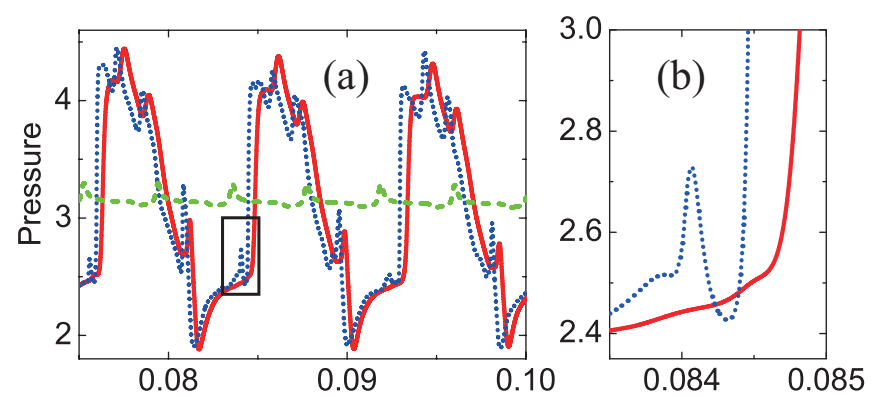

FIG. 12. Evolution of the shock pressure. The rectangle in panel (a) is amplified in panel (b). The solid line denotes the 2D detonation, the dotted line denotes the $2 \mathrm{D}$ detonation without nonequilibrium effects, and the dashed line denotes the 1D detonation.

the start and end of a period of the unsteady detonation, and the period is $8.6 \times 10^{-3}$. An arrow points to the reaction layer in the left column, which shows convex or concave shapes. In the middle column, an arrow is located at the preshock front consisting of an incident wave and a Mach stem, and the other arrow labels a secondary Mach stem behind. The incident wave is close to the location of maximum pressure, while the Mach stem is relatively far. The point of maximum (or minimum) pressure alternates from the center to the $y$ boundary. The above three arrows are plotted in the right column as well. Comparing the three fields, we can find that the nonequilibrium effects are pronounced where gradients of the reaction process and pressure are large. Specifically there are significant nonequilibrium effects around the preshock wave and the reaction zone. In addition, the nonequilibrium effects are strong around the transmitted wave after the detonation front. From the contours of $\Delta$, we could see clearly the evolution of detonation cellular structures.

To further investigate the nonequilibrium effects on detonation, we simulate a 2D unsteady detonation ignoring nonequilibrium effects, and compare it to the one considering nonequilibrium effects. In addition, a 1D unsteady detonation with nonequilibrium effects is simulated. All parameters are identical to those in Fig. 11. Figure 12 illustrates the evolution of the shock pressure in the above three cases. Obviously the 2D unsteady detonations with and without nonequilibrium effects are similar in amplitude and period, which are about 2.4 and 0.009 , respectively. Although the overall behaviors of the 2D detonations with and without nonequilibrium effects are similar, the details are quite different. As shown in Fig. 12(b), the detonation with nonequilibrium effects has a smooth profile because the nonequilibrium effects dissipate and eliminate small structures of fluid flows. Moreover, the amplitude and period of the 1D detonation are 0.19 and 0.004 , respectively. The amplitude and period of the $1 \mathrm{D}$ detonation are smaller than those of the 2D detonation, because the movements perpendicular to the propagation direction of the 2D detonation amplify the amplitude and lengthen the period.

\section{CONCLUSION}

We have presented a multiple-relaxation-time DBM for subsonic, sonic, and supersonic flows, under the influence of the chemical reaction and external force. Both specific heat ratio and Prandtl number are adjustable. This model could not only recover NS equations, but also measure various thermodynamic nonequilibrium effects beyond NS equations. The unified formulation for hydrodynamic and thermodynamic quantities is solved via the DBM. With utilization of a matrix inversion method, the collision, reaction, and force terms in the discrete Boltzmann equation are specified in a uniform way, which has high computational efficiency, physical accuracy, and convenience of coding. The capability of the DBM is demonstrated with benchmarks of chemical reactions in the free falling process, sound wave, thermal Couette flow, and steady and unsteady detonation. Furthermore, this model is employed to investigate nonequilibrium effects on unsteady detonation. It is worth noting that to our knowledge this is the first time that the DBM has been applied to 2D unsteady nonequilibrium detonations. Additionally, quantitative differences due to nonequilibrium effects have been probed in unsteady combustion. Significantly, it is demonstrated that the nonequilibrium effects suppress the detonation instability and dissipate small oscillations of fluid flows.

\section{ACKNOWLEDGMENTS}

This work is supported by the Natural Science Foundation of China (NSFC) under Grants No. 91441120 and 51806116, the China Postdoctoral Science Foundation under Grant No. 2017M620757, and the Center for Combustion Energy at Tsinghua University. Support from the UK Engineering and Physical Sciences Research Council under the project UK Consortium on Mesoscale Engineering Sciences (UKCOMES) (Grants No. EP/L00030X/1 and EP/R029598/1) is also gratefully acknowledged.

\section{APPENDIX A}

The matrix $\mathbf{M}_{\mathbf{f}}^{\mathrm{eq}}$ takes the form

$$
\mathbf{M}_{\mathbf{f}}^{\mathrm{eq}}=\left(\begin{array}{llll}
M_{f 1}^{\mathrm{eq}} & M_{f 2}^{\mathrm{eq}} & \cdots & M_{f 16}^{\mathrm{eq}}
\end{array}\right)^{\mathrm{T}},
$$

with elements $M_{f 1}^{\mathrm{eq}}=\rho, M_{f 2}^{\mathrm{eq}}=\rho u_{x}, \hat{f}_{3}^{\mathrm{eq}}=\rho u_{y}, M_{f 4}^{\mathrm{eq}}=$ $\rho\left[(D+I) T+u^{2}\right], M_{f 5}^{\mathrm{eq}}=\rho\left(T+u_{x}^{2}\right), M_{f 6}^{\mathrm{eq}}=\rho u_{x} u_{y}$, $M_{f 7}^{\mathrm{eq}}=\rho\left(T+u_{y}^{2}\right), M_{f 8}^{\mathrm{eq}}=\rho u_{x}\left[(D+I+2) T+u^{2}\right], M_{f 9}^{\mathrm{eq}}=$ $\rho u_{y}\left[(D+I+2) T+u^{2}\right], M_{f 10}^{\mathrm{eq}}=3 \rho u_{x} T+\rho u_{x}^{3}, \quad M_{f 11}^{\mathrm{eq}}=$ $\rho u_{y} T+\rho u_{x}^{2} u_{y}, \hat{f}_{12}^{\mathrm{eq}}=\rho u_{x} T+\rho u_{x} u_{y}^{2}, M_{f 13}^{\mathrm{eq}}=3 \rho u_{y} T+$ $\rho u_{y}^{3}, M_{f 14}^{\mathrm{eq}}=\rho\left[(D+I+2) T+u^{2}\right]+\rho u_{x}^{2}[(D+I+4)$ $\left.T+u^{2}\right], M_{f 15}^{\mathrm{eq}}=\rho u_{x} u_{y}\left[(D+I+4) T+u^{2}\right], M_{f 16}^{\mathrm{eq}}=$ $\rho\left[(D+I+2) T+u^{2}\right]+\rho u_{y}^{2}\left[(D+I+4) T+u^{2}\right]$.

The matrix $\mathbf{M}_{\mathbf{R}}$ is expressed by

$$
\mathbf{M}_{\mathbf{R}}=\left(\begin{array}{llll}
M_{R 1} & M_{R 2} & \cdots & M_{R 16}
\end{array}\right)^{\mathrm{T}},
$$

whose elements are $M_{R 1}=0, M_{R 2}=0, M_{R 3}=0, M_{R 4}=$ $\rho(D+I) T^{\prime}, \quad M_{R 5}=\rho T^{\prime}, M_{R 6}=0, M_{R 7}=\rho T^{\prime}, \quad M_{R 8}=$ $(D+I+2) \rho u_{x} T^{\prime}, M_{R 9}=(D+I+2) \rho u_{y} T^{\prime}, \quad M_{R 10}=$ $3 \rho u_{x} T^{\prime}, M_{R 11}=\rho u_{y} T^{\prime}, \quad M_{R 12}=\rho u_{x} T^{\prime}, M_{R 13}=3 \rho u_{y} T^{\prime}$, $M_{R 14}=\rho\left[2 T(D+I+2)+(D+I+5) u_{x}^{2}+u_{y}^{2}\right] T^{\prime}, \quad M_{R 15}=$ $\rho u_{x} u_{y}(D+I+4) T^{\prime}, M_{R 16}=\rho\left[2 T(D+I+2)+u_{x}^{2}+(D+\right.$ $\left.I+5) u_{y}^{2}\right] T^{\prime}$. 
The matrix $\mathbf{M}_{\mathbf{F}}$ reads

$$
\mathbf{M}_{\mathbf{F}}=\left(\begin{array}{llll}
M_{F 1} & M_{F 2} & \cdots & M_{F 16}
\end{array}\right)^{\mathrm{T}},
$$

with elements $M_{F 1}=0, M_{F 2}=\rho a_{x}, M_{F 3}=\rho a_{y}, M_{F 4}=$ $2 \rho a_{x} u_{x}+2 \rho a_{y} u_{y}, M_{F 5}=2 \rho a_{x} u_{x}, M_{F 6}=\rho a_{x} u_{y}+\rho a_{y} u_{x}$, $M_{F 7}=2 \rho a_{y} u_{y}, M_{F 8}=3 \rho a_{x} u_{x}^{2}+\rho a_{x} u_{y}^{2}+\rho a_{x}(D+I+$ 2) $T+2 \rho a_{y} u_{x} u_{y}, M_{F 9}=2 \rho a_{x} u_{x} u_{y}+3 \rho a_{y} u_{y}^{2}+\rho a_{y} u_{x}^{2}+$ $\rho a_{y}(D+I+2) T, \quad M_{F 10}=3 \rho a_{x}\left(u_{x}^{2}+T\right), \quad M_{F 11}=$ $2 \rho a_{x} u_{x} u_{y}+\rho a_{y}\left(u_{x}^{2}+T\right), \quad M_{F 12}=\rho a_{x}\left(u_{y}^{2}+T\right)+2 \rho a_{y} u_{x}$ $u_{y}, \quad M_{F 13}=3 \rho a_{y}\left(u_{y}^{2}+T\right), \quad M_{F 14}=2 \rho u_{x} a_{x}\left[2 u_{x}^{2}+u_{y}^{2}+\right.$ $(D+I+5) T]+2 \rho u_{y} a_{y}\left(u_{x}^{2}+T\right), M_{F 15}=\rho a_{x} u_{y}\left[3 u_{x}^{2}+\right.$ $\left.u_{y}^{2}+(D+I+4) T\right]+\rho a_{y} u_{x}\left[u_{x}^{2}+3 u_{y}^{2}+(D+I+4) T\right]$, $M_{F 16}=2 \rho a_{x} u_{x}\left(u_{y}^{2}+T\right)+2 \rho a_{y} u_{y}\left[u_{x}^{2}+2 u_{y}^{2}+(D+I+\right.$ 5) $T$ ].

The matrix $\mathbf{C}$ is specified as

$$
\mathbf{C}=\left(\begin{array}{llll}
\mathbf{C}_{1} & \mathbf{C}_{2} & \cdots & \mathbf{C}_{16}
\end{array}\right)^{\mathrm{T}},
$$

containing the blocks $\mathbf{C}_{i}=\left(\begin{array}{llll}C_{i 1} & C_{i 2} & \cdots & C_{i 16}\end{array}\right)$, with elements $C_{1 i}=1, C_{2 i}=v_{i x}, C_{3 i}=v_{i y}, C_{4 i}=v_{i}^{2}+\eta_{i}^{2}, C_{5 i}=$ $v_{i x}^{2}, C_{6 i}=v_{i x} v_{i y}, C_{7 i}=v_{i y}^{2}, C_{8 i}=\left(v_{i}^{2}+\eta_{i}^{2}\right) v_{i x}, C_{9 i}=$ $\left(v_{i}^{2}+\eta_{i}^{2}\right) v_{i y}, C_{10 i}=v_{i x}^{3}, C_{11 i}=v_{i x}^{2} v_{i y}, C_{12 i}=v_{i x} v_{i y}^{2}, C_{13 i}=$ $v_{i y}^{3}, C_{14 i}=\left(v_{i}^{2}+\eta_{i}^{2}\right) v_{i x}^{2}, C_{15 i}=\left(v_{i}^{2}+\eta_{i}^{2}\right) v_{i x} v_{i y}, C_{16 i}=$ $\left(v_{i}^{2}+\eta_{i}^{2}\right) v_{i y}^{2}$.

\section{APPENDIX B}

Via Chapman-Enskog analysis, it can be proved that the DBM could recover the following NS equations:

$$
\begin{gathered}
\frac{\partial \rho}{\partial t}+\nabla \cdot(\rho \mathbf{u})=0, \\
\frac{\partial(\rho \mathbf{u})}{\partial t}+\nabla \cdot(\rho \mathbf{u u}+\mathbf{P}+p \mathbf{I})=\rho \mathbf{a}, \\
\frac{\partial \xi}{\partial t}+\nabla \cdot(\xi \mathbf{u}+p \mathbf{u}+\mathbf{P} \cdot \mathbf{u}-\kappa \cdot \nabla T)=\rho \mathbf{a} \cdot \mathbf{u}+\rho \lambda^{\prime} Q,
\end{gathered}
$$

where $\mathbf{I}$ is an identity matrix, $\mathbf{P}$ is a second-order tensor with elements $P_{x x}=M_{f 5}^{\text {neq }}, P_{x y}=P_{y x}=M_{f 6}^{\text {neq }}$, and $P_{y y}=$ $M_{f 7}^{\text {neq }}, \kappa=\operatorname{diag}\left(\kappa_{x} \quad \kappa_{y}\right)$ is the heat conductivity with $\kappa_{x}=$ $(D+I+2) p /\left(2 S_{8}\right)$ and $\kappa_{y}=(D+I+2) p /\left(2 S_{9}\right)$. The pressure is $p=\rho T$, and the total energy is $\xi=(D+$ I) $\rho T / 2+\rho \mathbf{u} \cdot \mathbf{u} / 2$.

Moreover, the dynamic viscosity and thermal conductivity take the form $\mu=p / S_{\mu}$ and $\kappa=(D+I+2) p /\left(2 S_{\kappa}\right)$, under the conditions $S_{5}=S_{6}=S_{7}=S_{\mu}$ and $S_{8}=S_{9}=S_{\kappa}$. Then the elements of $\mathbf{P}$ become

$P_{\alpha \beta}=-\mu\left(\frac{\partial u_{\alpha}}{\partial r_{\beta}}+\frac{\partial u_{\beta}}{\partial r_{\alpha}}-\frac{2}{D} \frac{\partial u_{\chi}}{\partial r_{\chi}} \delta_{\alpha \beta}\right)-\mu_{B} \frac{\partial u_{\chi}}{\partial r_{\chi}} \delta_{\alpha \beta}$,

where $\mu_{B}=\mu[2 / D-2 /(D+I)]$ represents the bulk viscosity. Correspondingly the Prandtl number reads $\operatorname{Pr}=S_{\kappa} / S_{\mu}$. Meanwhile the specific-heat ratio is $\gamma=$ $(D+I+2) /(D+I)$. Obviously both Pr and $\gamma$ are flexible.
[1] C. K. Law, Combustion Physics (Cambridge University Press, Cambridge, 2006).

[2] E. Nagnibeda and E. Kustova, Non-equilibrium Reacting Gas Flows: Kinetic Theory of Transport and Relaxation Processes (Springer, Berlin, 2009).

[3] W. Han, Y. Gao, and C. K. Law, Combust. Flame 176, 285 (2017).

[4] S. T. Parete-Koon, C. R. Smith, T. L. Papatheodore, and O. E. B. Messer, Frontiers Phys. 8, 189 (2013).

[5] Y. Mahmoudi, N. Karimi, R. Deiterding, and S. Emami, J. Propul. Power 30, 384 (2014).

[6] B. M. Haines, F. F. Grinstein, and J. D. Schwarzkopf, J. Turbulence 14, 46 (2013).

[7] O. V. Kunova and E. A. Nagnibeda, Chem. Phys. 441, 66 (2014).

[8] M. Bisi, G. Martalo, and G. Spiga, Comput. Math. Appl. 66, 1403 (2013).

[9] E. Kustova, E. Nagnibeda, G. Oblapenko, A. Savelev, and I. Sharafutdinov, Chem. Phys. 464, 1 (2016).

[10] E. G. Kolesnichenko and Y. E. Gorbachev, Appl. Math. Model. 37, 5304 (2013).

[11] D. Bruno and V. Giovangigli, Phys. Fluids 23, 093104 (2011).

[12] Y. Zhang, R. Qin, and D. R. Emerson, Phys. Rev. E 71, 047702 (2005).

[13] J. Meng, Y. Zhang, N. G. Hadjiconstantinou, G. A. Radtke, and X. Shan, J. Fluid Mech. 718, 347 (2013).
[14] L. Chen, Q. Kang, Q. Tang, B. A. Robinson, Y.-L. He, and W.-Q. Tao, Int. J. Heat Mass Transf. 85, 935 (2015).

[15] G. Falcucci, G. Amati, V. K. Krastev, A. Montessori, G. S. Yablonsky, and S. Succi, Chem. Eng. Sci. 166, 274 (2017).

[16] M. Ashna, M. H. Rahimian, and A. Fakhari, Phys. Rev. E 95, 053301 (2017).

[17] C. Lin, A. Xu, G. Zhang, and Y. Li, Combust. Flame 164, 137 (2016).

[18] C. Lin, K. H. Luo, L. Fei, and S. Succi, Sci. Rep. 7, 14580 (2017).

[19] J. H. Lee, J. C. Kim, W. C. Jeon, S. G. Cho, and S. K. Kwak, J. Phys. Chem. C 121, 6415 (2017).

[20] D. J. Economou, T. J. Bartel, R. S. Wise, and D. P. Lymberopoulos, IEEE Trans. Plasma Sci. 23, 581 (1995).

[21] H. Struchtrup, in Macroscopic Transport Equations for Rarefied Gas Flows (Springer, Berlin, 2005).

[22] Y. Gan, A. Xu, G. Zhang, Y. Zhang, and S. Succi, Phys. Rev. E 97, 053312 (2018).

[23] H. Struchtrup and M. Torrilhon, Phys. Fluids 15, 2668 (2003).

[24] Y. Qian and S. Orszag, J. Stat. Phys. 81, 237 (1995).

[25] S. Succi, G. Bella, and F. Papetti, J. Sci. Comput. 12, 395 (1997).

[26] H. Yu, L. S. Luo, and S. S. Girimaji, Int. J. Comput. Eng. Sci. 3, 73 (2002).

[27] K. Yamamoto, N. Takada, and M. Misawa, Proc. Comb. Inst. 30, 1509 (2005). 
[28] E. Chiavazzo, I. V. Karlin, A. N. Gorban, and K. Boulouchos, Int. J. Numer. Methods Heat Fluid Flow 21, 494 (2011).

[29] Q. Li, K. H. Luo, Q. J. Kang, Y. L. He, Q. Chen, and Q. Liu, Prog. Energy Combust. Sci. 52, 62 (2016).

[30] G. Wellein, T. Zeiser, G. Hager, and S. Donath, Comput. Fluids 35, 910 (2006).

[31] V. Novozhilov and C. Byrne, Numer. Heat Transf. A 63, 824 (2013).

[32] B. Yan, A. Xu, G. Zhang, Y. Ying, and H. Li, Frontiers Phys. 8, 94 (2013).

[33] C. Lin, A. Xu, G. Zhang, and Y. Li, Commun. Theor. Phys. 62, 737 (2014).

[34] C. Lin and K. H. Luo, Combust. Flame 198, 356 (2018).

[35] A. Xu, C. Lin, G. Zhang, and Y. Li, Phys. Rev. E 91, 043306 (2015).

[36] C. Lin and K. H. Luo, Comput. Fluids 166, 176 (2018).
[37] Y. Gan, A. Xu, G. Zhang, P. Zhang, and Y. Li, EPL 97, 44002 (2012).

[38] M. Bouzidi, D. d'Humieres, P. Lallemand, and L. S. Luo, J. Comput. Phys. 172, 704 (2001).

[39] H. D. Ng, M. I. Radulescu, A. J. Higgins, N. Nikiforakis, and J. H. S. Lee, Combust. Theory Model. 9, 385 (2005).

[40] Y. Zhang, A. Xu, G. Zhang, and Z. Chen, Commun. Theor. Phys. 69, 77 (2018).

[41] Y. Hu, D. Li, S. Shu, and X. Niu, Int. J. Heat Mass Transf. 104, 544 (2017).

[42] F. Chen, A. Xu, and G. Zhang, Front. Phys. 11, 114703 (2016).

[43] M. Watari, Physica A (Amsterdam) 382, 502 (2007).

[44] H. Zhang and F. Zhuang, Adv. Appl. Mech. 29, 193 (1991).

[45] See the Supplemental Material at http://link.aps.org/ supplemental/10.1103/PhysRevE.99.012142 for more details about unsteady detonations. 